\title{
THE FIRST CASE REPORT OF FATAL ACUTE PULMONARY DYSFUNCTION IN A SYSTEMIC SCLEROSIS PATIENT TREATED WITH RITUXIMAB THERAPY
}
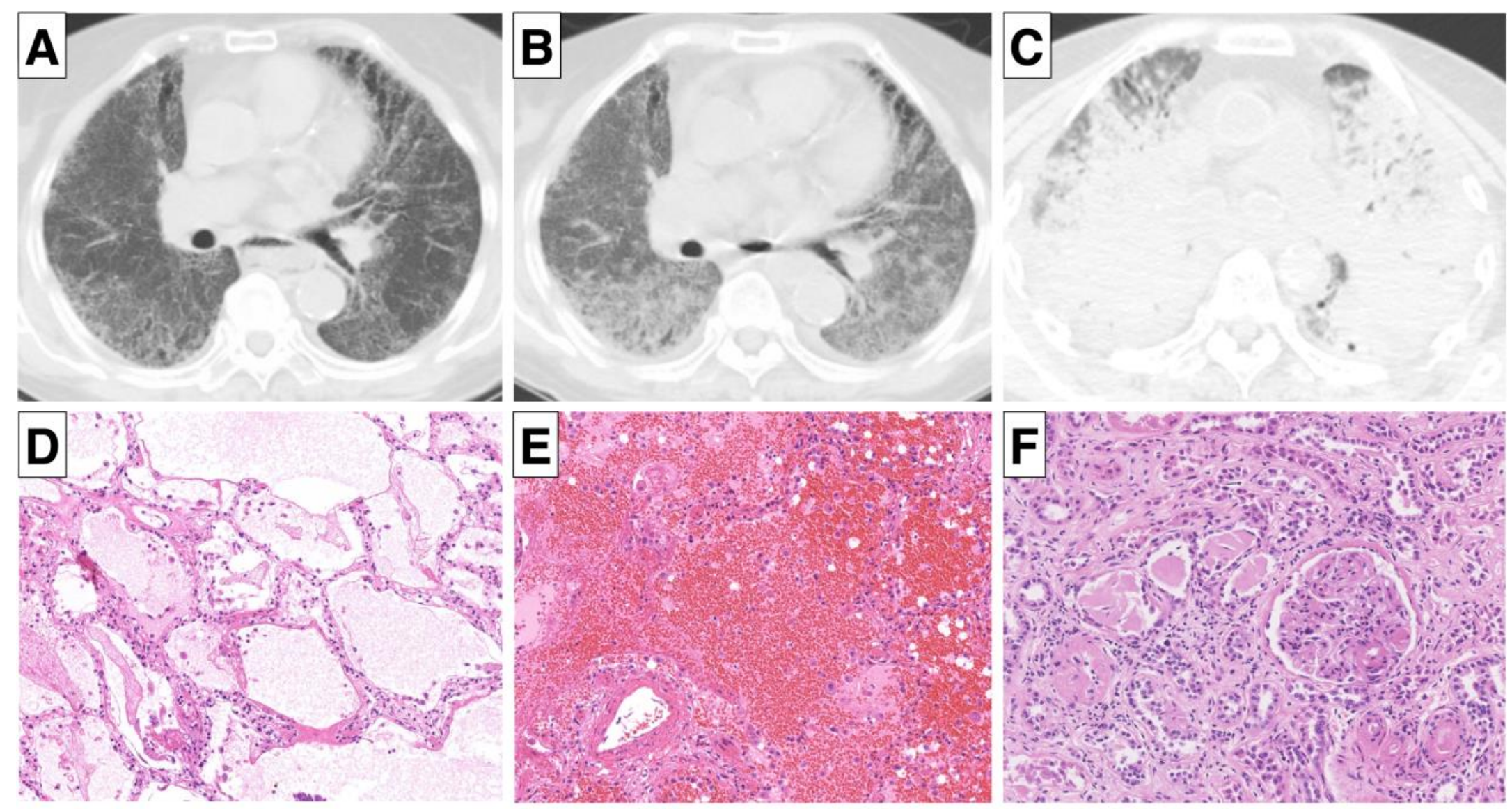

Supplementary Figure 1. The course of chest CT findings (A-C) and histopathological sections stained with hematoxylin and eosin (DF). (A) One day before the event of hemoptysis. Interstitial pattern compatible with SSc-ILD was found. (B) Three hours after the event of hemoptysis. Bilateral patchy ground-glass opacity was newly emerged. (C) Postmortem examination showed symmetric diffuse bilateral consolidation and increased pleural effusion. (D) Lung. Interstitial edema of the alveolar wall, dilatation of micro vessels, and hyaline membrane implied DAD. (x100) (E) Lung. Diffuse alveolar hemorrhage without capillaritis was found. (x100) (F) Kidney. Glomerular capillaries were obstructed by thrombi of platelets and fibrin, which was compatible with TMA. Platelets are confirmed with CD61 staining (not shown). There was no sign of glomerulonephritis. (x200) 

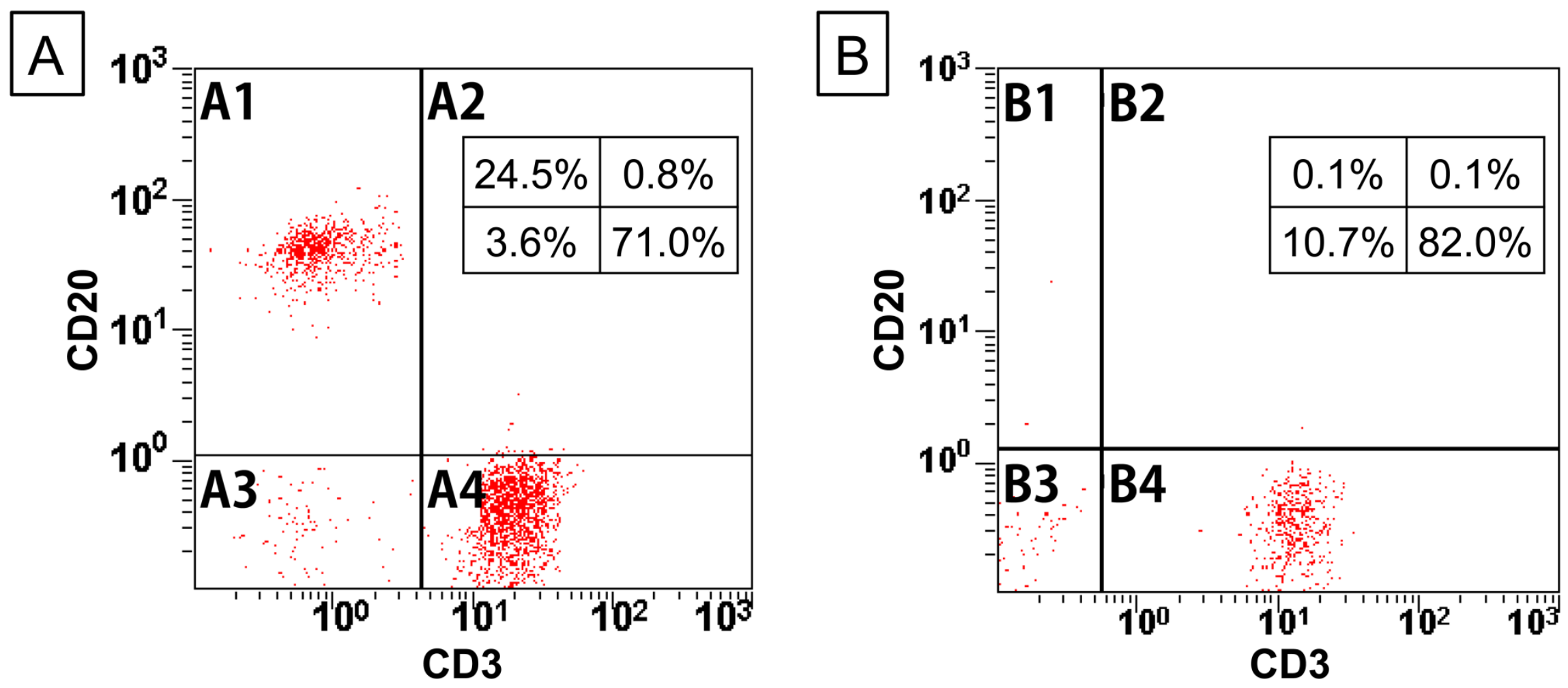

Supplementary Figure 2. Flow cytometric analysis of the patient's leukocytes that was decided by gating on CD45+ cells. (A) The percentage of CD20+CD3- is $24.5 \%$ before the treatment. (B) The percentage of CD20+CD3- is dropped to $0.1 \%$ in acute phase of the disease. 